ISSN: $2394-2258$

Available at http://scientificadvances.co.in

DOI: http://dx.doi.org/10.18642/ijamml_7100121727

\title{
SEGMENTATION OF MRI IMAGES VIA MATHEMATICAL MORPHOLOGY
}

\author{
T. Rahman ${ }^{\mathrm{a}}$, Jianqiang Gao ${ }^{\mathrm{b}}$ and Srinivas Nowduric \\ aDepartment of Computer Science and Engineering, Jahangirnagar University, \\ Dhaka, Bangladesh \\ ${ }^{b}$ College of Computer and Information, Hohai University, Nanjing, 210098, \\ P. R. China \\ 'Department of SS-LET, FSC, 2350 Braodhollow Road, Farmingdale NY 11735, \\ USA
}

\begin{abstract}
Magnetic resonance imaging (MRI) has become an emergent research area in medical field in finding the exact size, shape, position, appearance, and location of tumor. In this paper, we develop and implement a new method based on mathematical morphology, which can segment brain tumor from MRI images, usable for early detection of intracranial tumors. The method consists of two stages to detect and segment a brain tumor. Firstly, quality of input image is enhanced using morphological operators. Secondly, appropriate threshold value is used to segment brain tumor from MRI images. Experimental result confirms the superiority of the proposed approach in terms of performance preservation.
\end{abstract}

Keywords: magnetic resonance imaging (MRI), mathematical morphology, detection.

\footnotetext{
${ }^{*}$ Corresponding author.

E-mail address: dr.jq.gao@gmail.com (Jianqiang Gao) and nowdurs@farmingdale.edu (Srinivas Nowduri).
}

Copyright () 2016 Scientific Advances Publishers

2010 Mathematics Subject Classification: 68U10, 68U20.

Received October 5, 2016 


\section{Introduction}

Imaging has an essential role in the improvement of public health in all population groups. It comprises several different technologies that are used to visualize the anatomical structures of human body in order to diagnose, monitor or treat medical conditions. Each type of technology gives different information about different parts of human body being studied or treated. MRI is a medical imaging technology that uses radio waves and magnetic fields to produce detailed images of organs and tissues. It helps to clarify and distinguish the neural architecture of human body [1] and to detect brain tumor.

Brain tumors are abnormal and uncontrolled proliferations of cells. In fact, brain tumors are the second leading cause of cancer related deaths in children and young adults. The anatomy of the brain can be normally viewed by the MRI scan or CT scan. In this paper, the MRI scanned image is taken for the entire process. The MRI is more effective the CT scan for the diagnosis purpose as it does not use any radiation rather use magnetic field and radio waves [2]. Different type of algorithm were developed for the detection of brain tumor from MRI scan. Recent techniques achieved in researches for the detection of brain tumor can be classified as histogram based method, edge based segmentation, colour based segmentation, cohesion self merging based partition $K$-mean algorithm and so on [3].

Automatic segmentation of brain tumor is necessary due to the following regions:

(1) To delineate the boundaries of different tissues in order to determine difference between healthy versus diseased.

(2) MRI data is most relevant in the studies of head, specially for tracking the size of the brain tumor and other brain related problems [1].

(3) It also helps for early detection of intracranial tumors and precise estimation of tumor boundaries [1]. 
(4) Automatic segmentation process also helpful for the removal of the unnecessary information present in the MRI images.

(5) It is one of the key system for developing computer aided diagnosis systems.

(6) Tumor segmentation method also usable during surgical operations and monitoring treatment outcomes.

Recently, lots of algorithms have been proposed for segmentation process. Dynamic fuzzy clustering analysis [7], region growing method [8] and so on. In this paper, the main objective is to segment tumor region from MRI brain images, which is based on mathematical morphology for segmenting tumor regions from MRI images.

The remainder of this paper is organized as follows. Section 2 describes the methodology. Section 3 reports the experimental results. Section 4 concludes this paper.

\section{Methodology}

In medical field, MRI imaging plays a vital role by providing better diagnostic and therapeutic information. It is usually the technique of choice for tumor growth assessment, surgery planning and chemotherapy and radiotherapy follow up. The segmentation of the tumor allows to quantity the mass volume and response to the treatment, usually has to be performed manually, which is a time consuming and cumbersome activity [4]. This study presents a brain tumor segmentation method which purpose is to easily segment tumor masses in MRI images. A typical block diagram of our proposed approach is shown in Figure 1. The whole segmentation process is divided into two steps: (i) image enhancement; (ii) image segmentation.

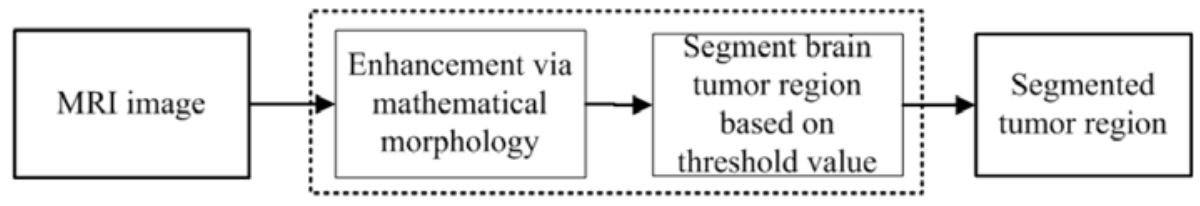

Figure 1. Block diagram of brain tumor segmentation. 


\subsection{Image enhancement}

In medical imaging enhancement is used to make image clearer, smoother and improves the quality of the input image. It can be accomplished by removing noise, enhancing contrast, modifying shapes and emphasizing edges [5]. It is widely applied in the field of radiology where image quality is important for diagnosis. Different types of tools have developed and applied to medical images for enhancement such as histogram modification, mean filters, Gaussian filters, Gabor filters, morphological filters etc. There are two methods based on morphological features for contrast enhancement. The first one is Top-Hat which deals images after segmentation process whereas other deals with contrast of the original image to enhance the segmentation process [5].

In image processing and analysis mathematical morphology is a relatively new approach. This approach is based on geometry. The basic idea is to probe an image with a template shape, which is called structuring element, to quantify the manner in which the structuring element fits within a given image [6]. In fact, the objects in an image are considered as sets of points and operations are defined between two sets, the objects and the structuring element (SE). All mathematical morphology operations are based on dilation and erosion. In mathematical morphology, we regard the pixel intensities as topographical highs. In this paper, we apply the following morphological operations to enhance the input image for further segmentation process:

$$
\begin{gathered}
\dot{x}=\left(x^{0} \circ r_{i} B_{D i s k}\right)=\left(x^{0}-x^{0} \circ r_{i} B_{D i s k}\right), \\
\ddot{x}=\left(\left(\dot{x} \bullet r_{i} B_{D i s k}\right) \oplus r_{j} B_{D i s k}\right) \circ r_{j} B_{D i s k} .
\end{gathered}
$$

In Equations (1) and (2), $\dot{x}$ is the input image. $r_{i} B_{D i s k}$ and $r_{j} B_{D i s k}$ are the disk type structuring element with radius $i$ and $j$, respectively. From the experimental results, we found that the radius $i=6$ and $j=2$ yields the best performance of the morphological algorithm. 


\subsection{Image segmentation}

After enhancement we need to segment tumor region from MRI scan. For this, we convert the enhanced image into binary image based on appropriate threshold value. The output binary image has values of 0 (black) for all pixels in the input image with luminance less than threshold value and 1 (white) for all other pixels. Experimentally, we found that 0.65 as threshold value gives better result for the segmentation process as converting gray to binary image. Thus, the output image will be the segmented tumor region from input MRI image.

\section{Experimental Results}

To evaluate the performance of proposed method for segmenting tumor region from MRI images, we used 15 images in our experiment. All the images were taken from web. Figure 2 shows some sample MRI images. The image quality will be enhanced and helpful for extraction process via using mathematical morphology. The enhanced images are shown in Figure 3 by using Equations (1) and (2). And then the results of segmentation process based on threshold value are shown in Figure 4.
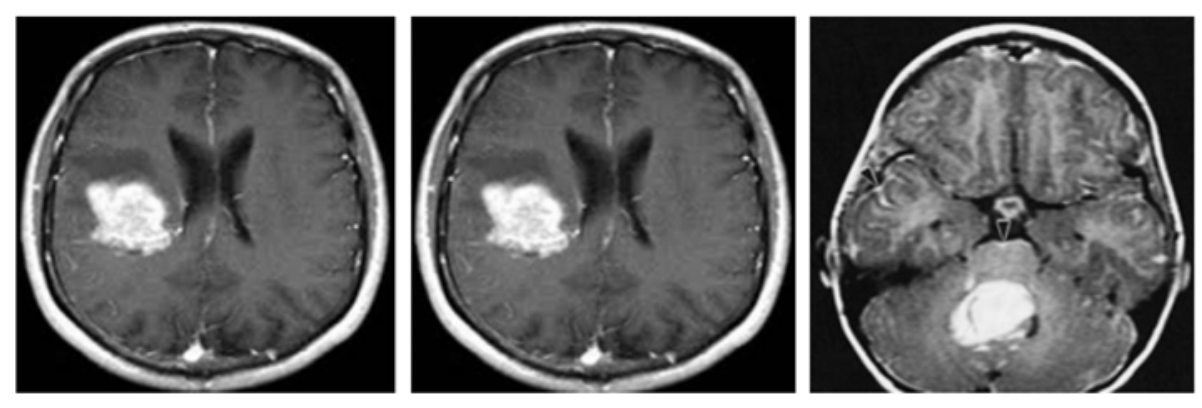

Figure 2. Input MRI images. 

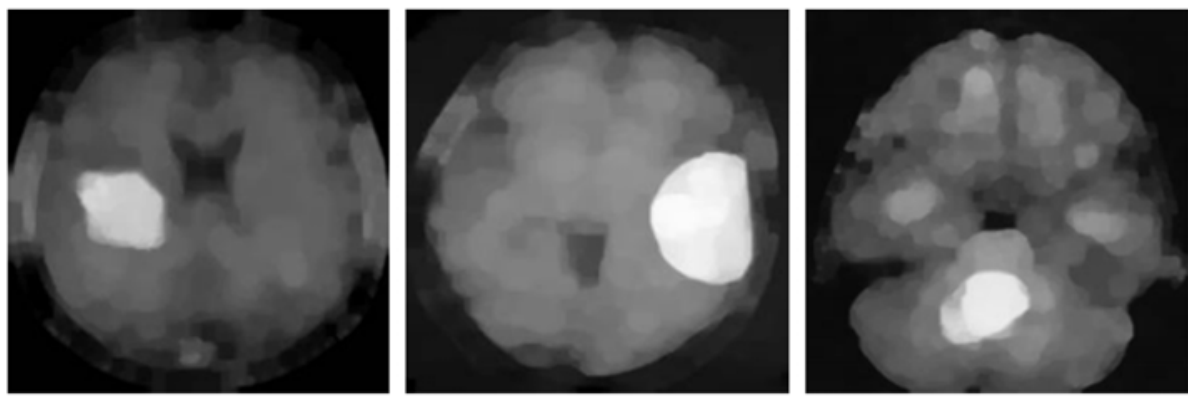

Figure 3. Enhanced image with morphological operation.
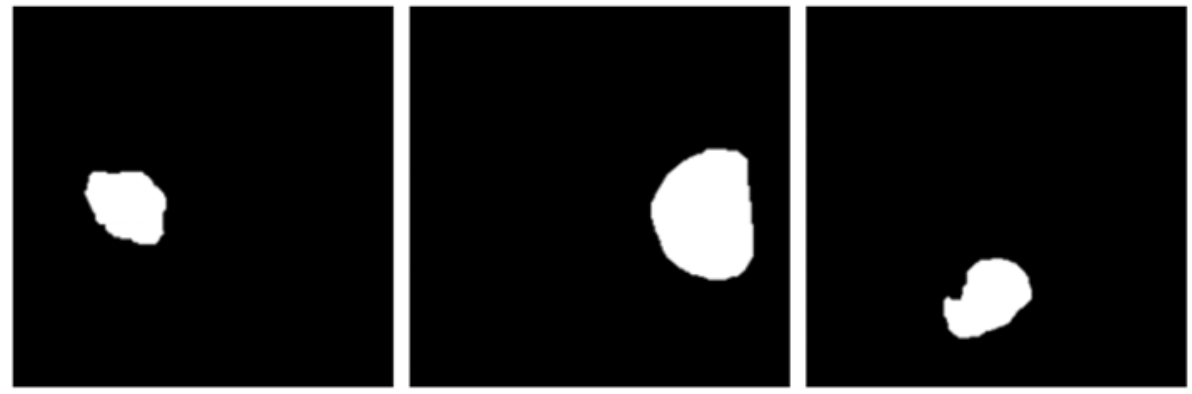

Figure 4. Segmented image with brain tumor.

Table 1 shows the result of segmentation performance using proposed method, which confirms the effectiveness of our technique.

Table 1. The result of proposed approach

\begin{tabular}{ll}
\hline Total number of sample image & 15 \\
Successfully segmented & 13 \\
Partially segmented & 1 \\
Failed & 1 \\
\hline
\end{tabular}

\section{Conclusion}

In this paper, a method of segmenting brain tumor regions from MRI image is discussed. The whole process is divided into two distinct stages: image enhancement and image segmentation. The experimental results 
also shown that the proposed approach is effective. The segmenting regions can be used further for many clinical operations and image analysis. The future concentrate to automate the determination of size and shape of brain tumor from MRI images.

\section{References}

[1] S. M. Ali, L. K. Abood and R. S. Abdoon, Brain tumor extraction in MRI images using clustering and morphological operations techniques, Int. J. Geographical Info. System Appl. Remote Sensing 4(1) (2013).

[2] R. Rana, H. S. Bhadauria and A. Singh, Study of various methods for brain tumor segmentation from MRI images, International Journal of Emerging Technology and Advanced Engineering 3(6) (2013), 338-342.

[3] R. G. Selkar and M. N. Thakare, Brain tumor detection and segmentation by using thresholding and watershed algorithm, International Journal of Advanced Information and Communication Technology 1(3) (2014), 321-324.

[4] M. de Carvalho Alegro, E. A. Junior and R. de Deus Lopes, Computerized brain tumor segmentation in magnetic resonance imaging, Scientific Electronic Library Online.

[5] A. Gade, R. Vig and V. Kulkarni, Segmentation of tumor region in MRI images of brain using mathematical morphology, International Journal of Image Processing (IJIP) 8(3) (2014), 95.

[6] Available: http:

homepages.inf.ed.ac.uk/rbf/CVonline/LOCALCOPIES/GASTERATOS/SOFT/2.htm

[7] X. Cheng and X. Gong, An image segmentation of fuzzy c-means clustering based on the combination of improved ant colony algorithm and genetic algorithm, Education Technology and Training, 2008 and 2008 International Workshop on Geoscience and Remote Sensing, ETT and GRS 2008, International Workshop on. IEEE 2 (2008), 804-808.

[8] W. Deng, W. Xiao, H. Deng et al., MRI brain tumor segmentation with region growing method based on the gradients and variances along and inside of the boundary curve, 3rd International Conference on Biomedical Engineering and Informatics. IEEE 1 (2010), 393-396. 